\title{
Safety Evaluation of Subchronic Oral Administration of Oleuropein and its Semisynthetic Peracetylated Derivative
}

\author{
Saverio Massimo Lepore ${ }^{1}$, Francesca Trimboli ${ }^{1}$, Marilena Celano ${ }^{1}$, Manuela Oliverio ${ }^{1}$, Roberta Fran- \\ cesca De Rose ${ }^{1}$, Giovanni Loprete ${ }^{1}$, Nicola Costa ${ }^{1}$, Carla Di Loreto ${ }^{2}$, Giuseppe Damante ${ }^{2}$, Sonia Bonac- \\ ci $^{1}$, Antonio Procopio ${ }^{1}$, Diego Russo ${ }^{1 *}$, Domenico Britti ${ }^{1}$

\begin{abstract}
${ }^{1}$ Department of Health Sciences, University “Magna Graecia” of Catanzaro, Campus "S. Venuta”, Viale Europa, Germaneto, 88100 Catanzaro, Italy

${ }^{2}$ Department of Medical and Biological Sciences, University of Udine, Piazzale Kolbe 4, 33100 Udine, Italy
\end{abstract}

"Corresponding author: Diego Russo, Professor, Department of Health Sciences, University "Magna Graecia" of Catanzaro, Campus “S. Venuta”, Viale Europa, 88100 Catanzaro, Italy, Tel: +3909613694124; Email: d.russo@unicz.it

\begin{abstract}
Olive leaf extracts are a natural source of phenolic compounds; this includes secoiridoid oleuropein (OLE), which has been widely investigated for its beneficial health effects. Previous in vitro studies have revealed antiproliferative and antioxidant activity in peracetylated oleuropein that is even stronger than oleuropein itself. The aim of the present study was to evaluate the effect of the subchronic administration of oleuropein, and for the first time, also evaluate its semisynthetic peracetylated derivative (peracetylated oleuropein, Ac-OLE). OLE and Ac-OLE were orally administered to male C57BL/6JOlaHsd mice at doses of $20 \mathrm{mg} / \mathrm{kg} /$ day for 15 weeks. In comparison to the control group, there were no treatment-related clinical signs of toxicity, nor were there any changes to note in body weight, food consumption, hematological and clinical chemistry parameters, or liver weight. Moreover, the histological examination of the liver, kidney, and lungs did not reveal any morphological alterations. These findings demonstrate that no adverse effects may be attributed to the subchronic oral administration of $20 \mathrm{mg} / \mathrm{kg} /$ day of Ac-OLE. However, additional experiments are necessary to clarify the action of the peracetylated compound in light of its therapeutic use.
\end{abstract}

Received date: November 25, 2015

Accepted date: February 15, 2016

Published date: February 18, 2016

Citation: Lepore, SM., et al. Safety Evaluation of Subchronic Oral Administration of Oleuropein and its Semisynthetic Peracetylated Derivative (2016) J Pharm Pharmaceutics 3(1): 40- 45.

DOI: $10.15436 / 2377-1313.16 .014$

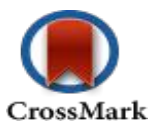

Keywords: Oleuropein; Peracetylated oleuropein; Dietary supplement; Subchronic toxicity

\section{Introduction}

Olive tree (Olea europaea) leaves have been widely used in traditional remedies in European and Mediterranean countries for centuries. They contain many potentially bioactive compounds that possess several pharmacological properties ${ }^{[1,2]}$. Among them, secoiridoid oleuropein (OLE) constitutes up to $6-9 \%$ of dry matter in the leaves. It is also present in considerable amounts in drupes, while its concentration in virgin olive oil is significantly reduced due to the enzymatic degradation that occurs during olive processing ${ }^{[1-4]}$. Due to their health benefits, oleuropein, oleuropein derivatives, and seco-phenolic compounds are all considered to be important elements that make extra virgin olive oil a staple part of the Mediterranean diet. Also, the compounds include the ability to scavenge reactive oxygen species $(\mathrm{ROS})^{[5-7]}$, to inhibit the proliferation of several tumor cell lines ${ }^{[5,8-11]}$, and also to protect against cardiovascular diseases ${ }^{[3,12-15]}$ and metabolic disorders as Diabetes mellitus ${ }^{[16-19]}$. In addition, they are effective in experimental models of age-related disorders, such as neurodegenerative diseases ${ }^{[14,20,21]}$. By using an innovative extractive method that employs renewable sources of primary matter and adopts sustainable synthetic strategies, we obtained OLE and some acetylated derivatives in good yields and very mild conditions ${ }^{[22,23]}$; interestingly, the latter compounds have shown an improved capacity to permeate the molecular membrane, in addition to their 'drug likeness ' ${ }^{\text {[22] }}$. Moreover, we have recently demonstrated that peracetylated oleuropein (Ac-OLE) possesses in vitro an antioxidant and a growth-inhibitory activity that is even stronger than OLE when acting against both breast and thyroid cancer cell lines ${ }^{[8,9]}$. While the absence of toxicity of oleuropein is well established ${ }^{[1,5,11]}$, very little is known about the in vivo effects of its peracetylated derivative. In this work, we investigated the comprehensive subchronic effects regarding the 
oral administration of Ac-OLE as compared to OLE in C57BL/ 6JOlaHsd mice.

\section{Materials and Methods}

\section{Study design}

Animal care was in compliance with Italian regulations regarding the protection of animals used for experimental and other scientific purposes (D.M. 116/92) as well as complying with the European Union regulations (O.J. of E.C. L 358/1 12/18/1986). All experiments were conducted in accordance with the Guide for the Care and Use of Laboratory Animals. All efforts were made to minimize the number of animals used in this study and their suffering.

\section{Compounds}

OLE and Ac-OLE were obtained as described ${ }^{[22]}$. Briefly, dried olive leaves from cultivar Coratina of O. Europaea were extracted in water under microwave-irradiation, and OLE was isolated as a pure compound from the aqueous extract after evaporation under reduced pressure and washing three times with acetone. Ac-OLE was achieved after the chemical modification of OLE following a patented synthetic method ${ }^{[23]}$, and was subsequently used for biological tests after chromatographic purification. OLE and its peracetylated derivative were extensively characterized by El, S.N., H-NMR and LC/ESI-MS, as previously described ${ }^{[22]}$.

\section{Animals}

4-week-old male C57BL/6JOlaHsd mice were purchased from Harlan Laboratories S.r.l. (Udine, Italy). Mice were placed in quarantine for 2 weeks in Animal Facility conditions and observed for clinical signs on daily basis. Then, they were divided into three groups ( $\mathrm{n}=5$ in each group) of equal average body weight. One group was used as control, while the other two were used as treatment groups. The average weight at the time of treatment was $19.4 \pm 1.2 \mathrm{~g}$ for all mice. All animals were housed in a temperature $\left(20-22^{\circ} \mathrm{C}\right)$ and humidity $(64 \%)$ controlled animal room and maintained on a 12:12 h light/dark cycle. The temperature and relative humidity were recorded continuously on a daily basis. Mice were fed with Teklad Global 18\% Protein Rodent Diet (Harlan Laboratories S.r.l). Both feed and water were provided ad libitum throughout the acclimatization and study periods.

Treatment, clinical examination, body weight, naso-anal length, girth waist, and feed consumption

Animal grouping was done using the method of body weight stratification and randomization. The grouping was done 1 day prior to the beginning of treatment. The body weight of mice after grouping was recorded and analyzed statistically for mean body weight to rule out the statistically significant difference. The group of control mice $(n=5$ each) was fed with a normal diet, while the other two groups of mice $(n=5$ each) were fed with the same diet with an additional administration of OLE and Ac-OLE $(20 \mathrm{mg} / \mathrm{kg} / \text { day })^{[12,15,16,18,27-29]}$ for 15 consecutive weeks ( 6 days a week).

Every day, OLE and Ac-OLE were freshly prepared from a condensed stock solution before administration and added to diet pellet; also, it was checked that each mouse had in- gested the food containing the daily dose of OLE and Ac-OLE. Water was used as a vehicle for the preparation of OLE, while corn oil was used as a vehicle for the preparation of Ac-OLE. Animals of all groups received the same vehicle used for the peracetylated compound and also had free access to food and water. The dose for each animal was calculated based on the weekly body weight of the mice. At the end of the treatment, all of the mice were sacrificed. Throughout the study period, the mice were observed daily for clinical signs of toxicity and mortality/morbidity. Body weight, naso-anal length and girth waist were measured weekly. To measure the total caloric intake, preweighed food was put into the cage and weighed a week later. Hematological, clinical chemistry and liver weighing were performed at termination. Histological examination was conducted on liver, lung, and kidney tissues from all groups.

\section{Blood collection}

Mice were fasted for $4 \mathrm{~h}$ before blood collection, and water was provided during the fasting period. Mice were deeply anesthetized with a combination of Tiletamine hypochloride and Zolazepam hypochloride (Zoletil, Virbac, France) at a dosage of $80 \mathrm{mg} / \mathrm{kg}$, and Medetomidine hydrochloride (Domitor, Orion Corporation, Espoo, Finland) at a dosage of $6.6 \mathrm{mg} / \mathrm{kg}$. This anesthetic was administered by intraperitoneal injection. Blood samples were obtained by cardiac venipuncture and were collected into blood collection tubes (Vacuette, Greiner Bio-One $\mathrm{GmbH}$, Kremsmunster, Austria) containing $\mathrm{K}_{3}$-EDTA.

\section{Hematology and Clinical chemistry}

Blood samples were analysed using a Hematology Analyzer ADVIA 2120 (Siemens Healthcare Diagnostics S.r.l, Milan, Italy) to obtain the following hematology parameters: White Blood Cells (WBC) and leukocyte formula (a percentage of neutrophils, lymphocytes, monocytes, and eosinophils), Red Blood Cells (RBC), Hemoglobin (HGB), Hematocrit (HCT), Mean Corpuscular Volume (MCV), Mean Corpuscular Hemoglobin $(\mathrm{MCH})$, Mean Corpuscular Hemoglobin Concentration (MCHC), and platelet count. Subsequently, the same blood samples were centrifuged at $1700 \mathrm{~g}$ for $10 \mathrm{~min}$ at room temperature to obtain plasma, before subsequently being stored at $-20^{\circ} \mathrm{C}$ until use. The levels of triglyceride, total cholesterol, low density lipoproteins (LDL), high density lipoproteins (HDL), alanine aminotransferase (ALT) and aspartate transaminase (AST) were determined using commercial reagents (Siemens Healthcare Diagnostics S.r.l.) on Dimension EXL (Siemens Healthcare Diagnostics S.r.1.), which is an automated biochemistry analyzer.

\section{Histological Study}

Immediately after sacrificing the mice, the liver, lung and kidney tissues were collected for histological studies. The whole liver was removed and weighed. The tissues were washed in a normal saline solution and immediately fixed in $10 \%$ neutral buffered formalin (Sigma-Aldrich S.r.l, Milan, Italy). The tissue samples were embedded in paraffin after dehydration through a graded ethanol series followed by xylene. Several sections (thickness 4-5 $\mu \mathrm{m}$ ) from each paraffin block were cut, mounted on slides and, after deparaffinization and rehydration, were stained with haematoxylin and eosin (H\&E) according to the standard methods for histological assessment under light microscopy. 


\section{Statistical analysis}

Statistical analyses were performed using Sigma Plot version 12 (Systat Software Inc.) on body weight, naso-anal length, girth waist, food consumption, energy intake, liver weight, and hematological and clinical chemistry parameters. The results are expressed as means \pm standard deviation (SD). A one-way ANOVA followed by Tukey multiple comparison tests was performed for different treatment groups and control group data. The differences between the groups were considered significant at $\mathrm{p}<0.01$.

\section{Results}

\section{Clinical signs, body weight, naso-anal length, girth waist and feed consumption}

There were no behavioral changes, clinical signs of toxicity, or mortality noted in any of the two groups of mice treated with OLE and Ac-OLE throughout the treatment period. The animal examination revealed a normal health status, and no abnormalities were observed during all the study period. Similarly, no significant differences were detected in body weight, naso-anal length, and girth waist measured during all the experimental periods among the groups (Table 1). The mean food consumption and energy intake were also comparable in all groups (Table 1).

Table 1: Effects of oleuropein (OLE) and peracetylated oleuropein (Ac-OLE) on body weight, naso-anal length, girth waist, food intake, and energy intake in C57BL/6JOlaHsd mice.

\begin{tabular}{|l|l|l|l|}
\hline & Control & OLE (20 mg/kg/day) & Ac-OLE (20 mg/kg/day) \\
\hline Initial body weight (g) & $20.6 \pm 1.1$ & $21.0 \pm 1.0$ & $20.2 \pm 1.3$ \\
\hline Final body weight (g) & $29.2 \pm 1.2$ & $30.3 \pm 1.3$ & $29.3 \pm 2.0$ \\
\hline Initial naso-anal length (cm) & $8.3 \pm 0.4$ & $8.9 \pm 0.2$ & $8.8 \pm 0.4$ \\
\hline Final naso-anal length (cm) & $9.9 \pm 0.1$ & $9.9 \pm 0.1$ & $9.9 \pm 0.1$ \\
\hline Initial girth waist (cm) & $6.6 \pm 0.1$ & $6.6 \pm 0.07$ & $6.3 \pm 0.1$ \\
\hline Final girth waist (cm) & $7.8 \pm 0.03$ & $7.7 \pm 0.3$ & $7.7 \pm 0.3$ \\
\hline Initial food intake (g/week) & $19.0 \pm 1.2$ & $18.2 \pm 1.3$ & $17.2 \pm 1.8$ \\
\hline Final food intake (g/week) & $20.8 \pm 0.8$ & $20.4 \pm 1.1$ & $20.0 \pm 1.2$ \\
\hline Initial energy intake (Kcal/week) & $58.9 \pm 3.8$ & $56.4 \pm 4.0$ & $53.3 \pm 5.5$ \\
\hline Final energy intake (Kcal/week) & $64.5 \pm 2.6$ & $63.2 \pm 3.5$ & $62.0 \pm 3.8$ \\
\hline
\end{tabular}

\section{Hematology}

There were no treatment-related toxicological signs in all of the hematology parameters analyzed in the blood samples collected at the end of the treatment period in any of the treated groups of mice (Table 2). Furthermore, we did not observe significant differences in the hematology parameters following the administration of OLE and Ac-OLE as compared to the control group in this strain of mice.

Table 2: Effects of oleuropein and peracetylated oleuropein on hematological parameters in C57BL/6JOlaHsd mice

\begin{tabular}{|l|c|c|c|c|c|}
\hline Parameter & Units & Control & OLE & Ac-OLE & Range \\
\hline WBC & $\left(10^{9} / \mathrm{L}\right)$ & $2.40 \pm 0.50$ & $2.20 \pm 0.80$ & $2.60 \pm 0.90$ & $2.30-7.70$ \\
\hline RBC & $\left(10^{12} / \mathrm{L}\right)$ & $8.60 \pm 1.10$ & $8.80 \pm 0.30$ & $8.40 \pm 0.40$ & $8.40-10.20$ \\
\hline HGB & $(\mathrm{mmol} / \mathrm{L})$ & $8.31 \pm 0.12$ & $8.25 \pm 0.37$ & $8.00 \pm 0.68$ & $7.80-9.00$ \\
\hline HCT & $($ fraction of $\%)$ & $0.42 \pm 0.06$ & $0.44 \pm 0.02$ & $0.42 \pm 0.03$ & $0.42-0.50$ \\
\hline MCV & $(\mathrm{fL})$ & $48.30 \pm 1.20$ & $49.60 \pm 1.10$ & $50.80 \pm 0.80$ & $48.00-52.00$ \\
\hline MCH & $(\mathrm{pg} / \mathrm{cell})$ & $14.60 \pm 0.10$ & $14.90 \pm 0.40$ & $15.30 \pm 0.70$ & $14.30-15.30$ \\
\hline MCHC & $(\mathrm{g} / \mathrm{L})$ & $304 \pm 7.50$ & $301 \pm 5.20$ & $302 \pm 13.20$ & $286-308$ \\
\hline Platelets & $\left(10^{9} / \mathrm{L}\right)$ & $1183.20 \pm 71.40$ & $1057.20 \pm 119.00$ & $1020.30 \pm 49.20$ & $980-1390$ \\
\hline Neutrophils & $(\%)$ & $7.40 \pm 1.80$ & $16.20 \pm 6.20$ & $6.70 \pm 2.70$ & $2-34$ \\
\hline Lymphocytes & $(\%)$ & $87.60 \pm 1.10$ & $80.60 \pm 5.60$ & $85.20 \pm 1.50$ & $59-97$ \\
\hline Monocytes & $(\%)$ & $0.80 \pm 0.20$ & $1.50 \pm 0.40$ & $1.10 \pm 0.20$ & 0 \\
\hline Eosinophils & $(\%)$ & $1.60 \pm 0.50$ & $2.30 \pm 0.10$ & $1.50 \pm 0.50$ & $0-2$ \\
\hline
\end{tabular}

Abbreviations: Ac-OLE = peracetylated oleuropein; HCT = hematocrit; HGB = hemoglobin; $\mathrm{MCH}=$ mean corpuscular hemoglobin; $\mathrm{MCV}=$ mean corpuscular volume; $\mathrm{MCHC}=$ mean corpuscular hemoglobin concentration; $\mathrm{OLE}=$ oleuropein; $\mathrm{RBC}=$ red blood cells; $\mathrm{WBC}=$ white blood cells.

\section{Clinical chemistry}

Table 3 shows the clinical chemistry parameters analyzed from the blood samples collected at the end of the treatment. There were no significant differences in both the triglyceride and total cholesterol levels in mice treated with OLE and Ac-OLE, 
as compared to control group. We found a significant difference $(p<0.01)$ between the control and treatment groups regarding the LDL fraction in OLE-; however, this was not the case in the Ac-OLE-treated mice. A significant increase in AST was noted in AcOLE-treated mice, which resulted in an approximately 2.5 fold difference, which was higher than those detected in the OLE-treated and control groups. However, the AST levels in this group of animals were still within the normal range of values expected for this strain of mice (Table 3).

Table 3: Effects of oleuropein and peracetylated oleuropein on serum chemistry parameters in C57BL/6JOlaHSD mice.

\begin{tabular}{|l|c|c|c|c|c|}
\hline Parameter & Units & Control & OLE & Ac-OLE & Range \\
\hline Cholesterol & $\mathrm{mmol} / \mathrm{L}$ & $2.81 \pm 0.16$ & $3.03 \pm 0.06$ & $2.79 \pm 0.16$ & $1.13-3.26$ \\
\hline HDL & $\mathrm{mmol} / \mathrm{L}$ & $2.58 \pm 0.23$ & $2.74 \pm 0.11$ & $2.48 \pm 0.24$ & $1.32-2.65$ \\
\hline LDL & $\mathrm{mmol} / \mathrm{L}$ & $0.36 \pm 0.07$ & $0.50 \pm 0.02 * *$ & $0.45 \pm 0.06$ & $0.36-0.80$ \\
\hline Triglycerides & $\mathrm{mmol} / \mathrm{L}$ & $1.16 \pm 0.23$ & $0.95 \pm 0.20$ & $1.30 \pm 0.11$ & $1.22-6.59$ \\
\hline ALT & $\mathrm{U} / \mathrm{L}$ & $36.50 \pm 4.80$ & $36.40 \pm 7.90$ & $39.30 \pm 1.50$ & $13.30-80.20$ \\
\hline AST & $\mathrm{U} / \mathrm{L}$ & $107.30 \pm 6.10$ & $134.80 \pm 23.80$ & $339.30 \pm 82.60 * *$ & $40.80-510.60$ \\
\hline
\end{tabular}

Abbreviations: Ac-OLE = peracetylated oleuropein; ALT = alanine aminotransferase; AST $=$ aspartate aminotransferase; HDL $=$ high density lipoproteins; LDL = low density lipoproteins; OLE = oleuropein. Reference ranges were calculated on the data published in Mouse Database Phenomena (Jackson Laboratory) (http:// phenome.jax.org) as mean $\pm 1.96 \mathrm{SD}$ except for ALT and AST, for which reference ranges were calculated as percentile $\left(2.5^{\text {th }}-97.5^{\text {th }}\right)$. $* *$ Statistically significant compared to the control group $(\mathrm{p}<0.01)$.

\section{Histopathology}

No changes were noted regarding liver weight among the groups following treatment with OLE and Ac-OLE (data not shown). As shown in (Figure 1), some sections of mouse liver revealed a preserved lobular structure; also, the liver cells showed nuclei of varied shapes and sizes. No other changes were observed. The lung and kidney tissues demonstrated a normal architecture in all the groups. Some proximal tubular cells in the kidneys showed vacuolization. No microscopic abnormalities were detected in any of the study groups (Figure 1).

\section{Control}

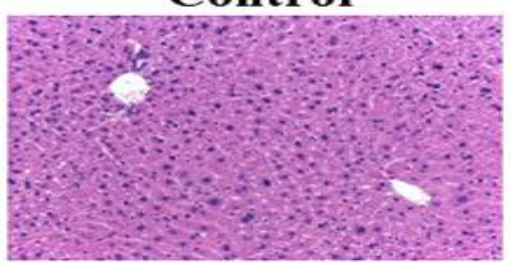

Lung
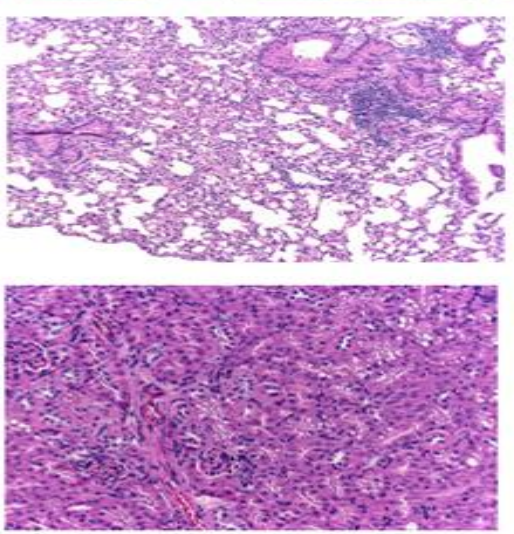

OLE
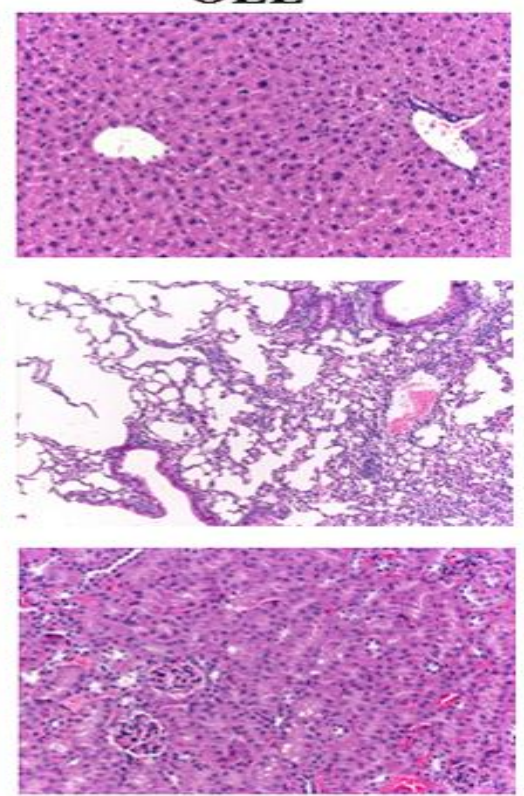

Ac-OLE
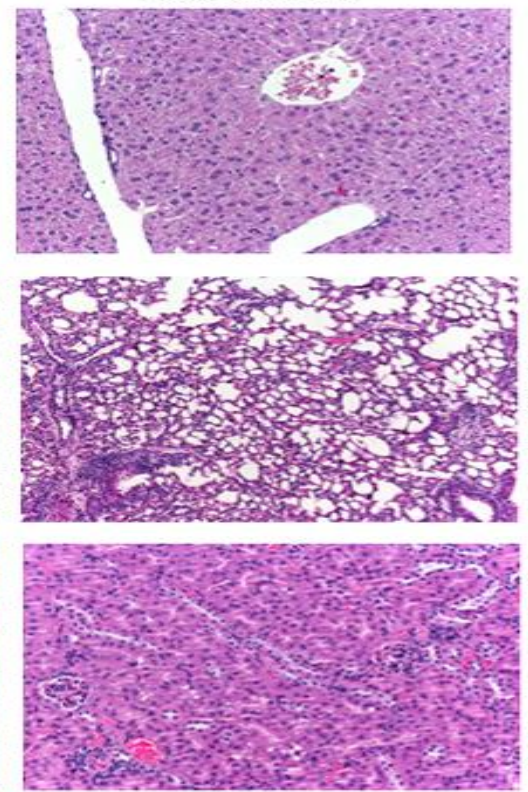

Figure 1: Effects of oleuropein (OLE) and peracetylated oleuropein (Ac-OLE) on liver, lung and kidney tissues. Top row. Sections showing the liver architecture and the components of basic liver lobules, with the portal area and the central venule. Haematoxylin and eosin, 20x. Middle row. Normal lung tissue is detected in all three groups. Haematoxylin and eosin, 10x. Bottom row. The kidney structure is preserved in all samples. Some tubular cells are vacuolated. Haematoxylin and eosin, 20x. No differences are present among the three study groups.

\section{Discussion}

In this study, we demonstrated that the oral administration to C57BL/6JOlaHsd male mice of oleuropein, and also, for the first time, its peracetylated derivative at levels up to $20 \mathrm{mg} / \mathrm{kg} / \mathrm{day}$ for 15 weeks was not associated with adverse effects. The analysis of the general conditions and appearance of the animals, or their growth, naso-anal length, girth waist, food consumption, hematology, clinical chemistry, liver weight, and histopathology clearly showed that OLE and Ac-OLE do not interfere with the normal metabolism of animals, as revealed by the absence of any significant differences in the above mentioned parameters. The only significant difference $(\mathrm{p}<0.01)$ observed between the control and treatment groups regards the LDL fraction in OLE-, though this is 
not the case in the Ac-OLE-treated mice. An increase of this parameter was noted when compared to the control animals group; however, the values remain within the normal laboratory ranges. Also, the serum levels of AST resulted in a significant increase in the Ac-OLE-treated mice; again, they were still within the normal range, so these changes may be considered as incidental biological variations and not treatment-related adverse effects. Such conclusions are supported by the histological analysis, which confirmed the absence of any abnormalities in the tissues of all of the treated animals. To the best of our knowledge, this is the first report regarding the influence of OLE and its peracetylated derivative treatment in vivo on hematology parameters. Both OLE and Ac-OLE did not induce any variation on mice hematology, which thus confirms the absence of subchronic toxicity for these two molecules. Recent reports ${ }^{[24]}$ have suggested the potential improvements concerning the OLE effect by protecting the molecule through its peracetylation. In previous studies, we have shown that Ac-OLE has a stronger anti-proliferative effect than OLE in the experimental models of tumor cells in vitro ${ }^{[8,9]}$. Also in a very recent study, we demonstrate the beneficial effects of Ac-OLE at the same dose $(20 \mathrm{mg} / \mathrm{kg} /$ day $)$ in the prevention of hepatic steatosis, hyperinsulinemia and weight gain in mice fed with high fat cafeteria diet ${ }^{[25]}$.

Such a chemical modification has been previously demonstrated to improve its permeability ${ }^{[22]}$, while still maintaining the biological activity of the parent compound; this is probably owed to the extensive deacetylation by carboxylesterases. Deacetylation can take place either within the cell, upon absorption of the acetylated molecule, or in the extracellular space created by the secreted esterases ${ }^{[26]}$. In addition, Procopio et al. ${ }^{[22]}$ have demonstrated the increased anti-inflammatory activity of Ac-OLE as compared to OLE both in vitro as a COX1 and COX-2 inhibitor, and also in vivo as a protecting agent against the carrageenan-induced paw edema in mice. Looking at these data as a whole thus suggests the potentiality to take advantage of the properties of this compound, by using it as additive in fatty foods or beverages. In conclusion, the present findings demonstrate that peracetylated oleuropein at the dose studied herein does not produce any toxic signs or clinically evident symptoms following subchronic oral administration. In the animals treated with Ac-OLE, there were no remarkable histological signs, or blood and serum chemical alterations. The effects of administering varied dosages of Ac-OLE will be analyzed in further studies in view of the potential use of this compound. Therefore, our preliminary results suggest promising alternatives for exploring the therapeutic and pharmacological use of this new molecule, as well as the investigation of its use as an additive for fatty functional foods.

\section{Acknowledgments}

This work was supported by a donation in the memory of Carmelo Stillitani to the UMG Foundation. Saverio Massimo Lepore is supported by a co-financed grant from the European Commission, the European Social Fund, and the Calabria Region. English has been edited by FirstEditing.com

\section{Conflict of interest}

The authors declare that they have no conflict of interest.

\section{References}

1. El, S.N., Karakaya, S. Olive tree (Olea europaea) leaves: Potential beneficial effects on human health. (2009) Nutr Rev 67(11): 632-638.

2. Omar, S.H. Oleuropein in olive and its pharmacological effects. (2010) Sci Pharm 78(2): 133-154.

3. Bulotta, S., Celano, M., Lepore, S.M., et al. Beneficial effects of the olive oil phenolic components oleuropein and hydroxytyrosol: focus on protection against cardiovascular and metabolic diseases. (2014) J Transl Med 12: 219.

4. Tripoli, E., Giammanco, M., Tabacchi, G., et al. The phenolic compounds of olive oil: structure, biological activity and beneficial effects on human health. (2005) Nutr Res Rev 18(1): 98-112.

5. Cicerale, S., Lucas, L., Keast, R. Biological activities of phenolic compounds present in virgin olive oil. (2010) Int J Mol Sci 11(2): 458479.

6. Cicerale, S., Lucas, L.J. Keast, R.S. Antimicrobial, antioxidant and anti-inflammatory phenolic activities in extra virgin olive oil. (2012) Curr Opin Biotechnol 23(2): 129-135.

7. Visioli, F., Poli, A., Gall, C. Antioxidant and other biological activities of phenols from olives and olive oil. (2002) Med Res Rev 22(1): 65-75.

8. Bulotta, S., Corradino, R., Celano, M., et al. Antioxidant and antigrowth action of peracetylated oleuropein in thyroid cancer cells. (2013) J Mol Endocrinol 51(1): 181-189.

9. Bulotta, S., Corradino, R., Celano, M., et al. Antiproliferative and antioxidant effects of oleuropein and its semisynthetic peracetylated derivatives on breast cancer cells. (2011) Food Chem 127(4): 1609-1614. 10. Goulas, V., Exarchou, V., Troganis, A.N., et al. Phytochemicals in olive-leaf extracts and their antiproliferative activity against cancer and endothelial cells. (2009) Mol Nutr Food Res 53(5): 600-608.

11. Hamdi, H.K., Castellon, R. Oleuropein, a non-toxic olive iridoid, is an anti-tumor agent and cytoskeleton disruptor. (2005) Biochem Biophys Res Commun 334(3): 769-778.

12. Andreadou, I., Iliodromitis, E.K., Mikros, E., et al. The olive constituent oleuropein exhibits anti-ischemic, antioxidative, and hypolipidemic effects in anesthetized rabbits. (2006) J Nutr 136(8): 2213-2219.

13. Carluccio, M.A., Siculella, L., Ancora, M.A., et al. Olive oil and red wine antioxidant polyphenols inhibit endothelial activation: antiatherogenic properties of Mediterranean diet phytochemicals. (2003) Arterioscler Thromb Vasc Biol 23(4): 622-629.

14. Omar, S.H. Cardioprotective and neuroprotective roles of oleuropein in olive. (2010) Saudi Pharm J 18(3): 111-121.

15. Andreadou, I., Benaki, D., Efentakis, P., et al. The natural olive constituent oleuropein induces nutritional cardioprotection in normal and cholesterol-fed rabbits: comparison with preconditioning. (2015) Planta Med 81(8): 655-663.

16. Al-Azzawie, H.F., Alhamdani, M.S. Hypoglicemic and antioxidant effect of oleuropein in alloxan-diabetic rabbits. (2006) Life Sci 78(12): 1371-1377.

17. de Bock, M., Derraik, J.G., Brennan, C.M., et al. Olive (Olea europaea L.) leaf polyphenols improve insulin sensitivity in middle-aged overweight men: a randomized, placebo-controlled, crossover trial. (2013) PLoS One 8(3): e57622.

18. Jemai, H., El Feki, A., Sayadi, S. Antidiabetic and antioxidant effects of hydroxytyrosol and oleuropein from olive leaves in alloxan-diabetic rats. (2009) J Agric Food Chem 57(19): 8798-8804.

19. Poudyal, H., Campbell, F., Brown, L. Olive leaf extract attenuates cardiac, hepatic, and metabolic changes in high carbohydrate, high fatfed rats. (2010) J Nutr 140(5): 946-953.

20. Daccache, A., Lion, C., Sibille, N., et al. Oleuropein and derivatives from olives as Tau aggregation inhibitors. (2011) Neurochem Int 58(6): 700-707.

21. Khalatbary, A.R., Ahmadvand, H. Neuroprotective effect of oleuropein following spinal cord injury in rats. (2012) Neurol Res 34(1): 44-51. 
22. Procopio, A., Alcaro, S., Nardi, M., et al. Synthesis, biological evaluation, and molecular modeling of oleuropein and its semisynthetic derivatives as cyclooxygenase inhibitors. (2009) J Agric Food Chem 57(23): 11161-11167.

23. Procopio, A., Sindona, G., Gaspari M., et al. Chemical catalytic method for the peracylation of oleuropein and its products of hydrolysis. (2008) International Patent.

24. Bulotta, S., Oliverio, M., Russo D, et al. Biological Activityof Oleuropein and its Derivatives. (2013) In: Ramawat KG, Mérillon JM. Natural Products Berlin: Heidelberg Springer-Verla: 3605-3638.

25. Lepore, S.M., Morittu, V.M., Celano, M., et al. Oral administration of oleuropein and its semisynthetic peracetylated derivative prevents hepatic steatosis, hyperinsulinemia and weight gain in mice fed with high fat cafeteria diet. (2015) Int J Endocrinol 2015: 431453.
26. Mateos, R., Goya, L., Bravo, L. Metabolism of the olive oil phenols hydroxytyrosol, tyrosol, and hydroxytyrosyl acetate by human hepatoma HepG2 cells. (2005) J Agric Food Chem 53(26): 9897-9905.

27. Kim, Y., Choi, Y., Park, T. Hepatoprotective effect of oleuropein in mice: Mechanisms uncovered by gene expression profiling. (2010) Biotechnol J 5(9): 950-960.

28. Park, S., Choi, Y., Um, S.J., et al. Oleuropein attenuates hepatic steatosis induced by high-fat diet in mice. (2011) J Hepatol 54(5): 984993.

29. Kim, S.W., Hur, W., Li, T.Z., et al. Oleuropein prevents the progression of steatohepatitis to hepatic fibrosis induced by a high-fat diet in mice. (2014) Exp Mol Med 46: e92.
Ommega Online Publishers

Journal Title: Journal of Pharmacy \& Pharmaceutics(JPP)

Journal Short Name: J Pharm Pharmaceutics
Journal ISSN: 2377-1313

E-mail: pharmacoinformatics@ommegaonline.com

Website: www.ommegaonline.org 